\title{
FIRST ORDER DEFORMATIONS OF PAIRS OF A RATIONAL CURVE AND A HYPERSURFACE*
}

\author{
BIN WANG ${ }^{\dagger}$
}

\begin{abstract}
Let $X_{0}$ be a smooth hypersurface (not assumed generic) in projective space $\mathbf{P}^{n}, n \geq$ 3 over the complex numbers, and $C_{0}$ a smooth rational curve on $X_{0}$. We are interested in the deformations of the pair $C_{0}, X_{0}$. In this paper, we prove that if the first order deformations of the pair exist along certain first order deformations of the hypersurface $X_{0}$, then the twisted normal bundle
\end{abstract}

$$
N_{C_{0} / X_{0}}(1)=\left.N_{C_{0} / X_{0}} \otimes \mathcal{O}_{\mathbf{P}^{n}}(1)\right|_{C_{0}}
$$

is generated by global sections.

Key words. Rational curve, hypersurface, twisted normal bundle.

AMS subject classifications. $14 \mathrm{~J} 70$.

1. Introduction. Let $X_{0}$ be a smooth hypersurface in $\mathbf{P}^{n}$ over $\mathbb{C}$, and $C_{0}$ be a smooth rational curve on $X_{0}$. The main focus of this paper is on how the existence of the first order deformation of the pair $C_{0} \subset X_{0}$ affects the twisted normal bundle $N_{C_{0} / X_{0}}(1)$ of the rational curve. Previously, there are many works on pairs $C_{0}, X_{0}$ by Albano and Katz ([1]), Clemens ([3], [4]), Katz ([7]), Pacienza ([8]), Voisin ([9], [10]), etc.(there are many important papers missing from this list). In this paper, we add more results to this list. But one of main differences of this paper from others, which also turns out to be the main difficulty, is our weaker first order assumption (1.3) in theorem 1.2 below. Such an assumption is useful in understanding the deformations of the pair. Deformations of pairs in more general setting were formulated and studied by Clemens ([5], [6]). Our results are not consequences of this study. To state the theorem in a precise way, let's give a formal description of the assumption. Throughout the paper varieties are over complex numbers. Let $H^{0}\left(\mathcal{O}_{\mathbf{P}^{n}}(h)\right)$ denote the vector space of homogeneous polynomials of degree $h=\operatorname{deg}\left(X_{0}\right)$ in $n+1$ variables for $n \geq 3$. Let $f_{0} \in H^{0}\left(\mathcal{O}_{\mathbf{P}^{n}}(h)\right)$ such that

$$
X_{0}=\operatorname{div}\left(f_{0}\right)
$$

is a smooth hypersurface. Let

$$
\left[f_{0}\right] \in \mathbf{P}\left(H^{0}\left(\mathcal{O}_{\mathbf{P}^{n}}(h)\right)\right)
$$

denote the corresponding point of $f_{0}$ in the projectivization. Let

$$
c_{0}: \mathbf{P}^{1} \rightarrow X_{0} \subset \mathbf{P}^{n}
$$

be an embedding of $\mathbf{P}^{1}$, whose image is $C_{0}$. Let

$$
\mathbb{H}^{1}\left(T_{X_{0}} \rightarrow N_{C_{0} / X_{0}}\right)
$$

be the hypercohomology of the complex, that is isomorphic to the tangent space of the deformation space of the pair $C_{0} \subset X_{0}$. Let $H^{1}\left(T_{X_{0}}\right)$ be cohomology group that

\footnotetext{
*Received September 14, 2012; accepted for publication September 28, 2012.

${ }^{\dagger}$ Mathematics and Computer Science Department, Rhode Island College, Providence, RI 02908, USA (bwang@ric.edu).
} 
is isomorphic to the tangent space of the deformation space of hypersurfaces at the point $X_{0}$. There is a known diagram ([1]):

$$
\begin{array}{ccc} 
& & \mathbb{H}^{1}\left(T_{X_{0}} \rightarrow N_{C_{0} / X_{0}}\right) \\
T_{\left[f_{0}\right]} \mathbf{P}\left(H^{0}\left(\mathcal{O}_{\mathbf{P}^{n}}(h)\right)\right) & \stackrel{\psi}{\rightarrow} & H^{1}\left(T_{X_{0}}\right)
\end{array}
$$

where the map $\psi$ is the differential at $\left[f_{0}\right]$ from $\mathbf{P}\left(H^{0}\left(\mathcal{O}_{\mathbf{P}^{n}}(h)\right)\right.$ to the deformation space of complex structures of the differential manifold of $X_{0}$. This diagram gives a relation between the first order deformations of the pair and the first order deformation of hypersurfaces at the level of moduli spaces (i.e. factoring out isomorphism). There is another version of $\phi$ without factoring out isomorphism (see (2.3) section 2).

Next we define a specific family of hypersurfaces.

Definition 1.1. Let $L_{i} \in H^{0}\left(\mathcal{O}_{\mathbf{P}^{n}}(1)\right), i=0, \cdots, h=\operatorname{deg}\left(X_{0}\right)$ be some non-zero sections (not assumed generic) that satisfy

$$
\left\{L_{i}=0\right\} \cap\left\{L_{j}=0\right\} \cap C_{0}=\emptyset, i \neq j .
$$

We consider the family of sections in $H^{0}\left(\mathcal{O}_{\mathbf{P}^{n}}(h)\right)$,

$$
F\left(a_{1}, \cdots, a_{h}, x\right)=f_{0}(x)+\sum_{i=0}^{h} a_{i} L_{0}(x) \cdots \hat{L}_{i}(x) \cdots L_{h}(x), \quad\left(\text { omit } L_{i}\right),
$$

parametrized by the coefficients $a_{i}, i=0, \cdots, h$. Since the degree $h$ hypersurfaces

$$
L_{0}(x) \cdots \hat{L}_{i}(x) \cdots L_{h}(x), i=0, \cdots, h
$$

are linearly independent over $\mathbb{C}, \mathbb{C}^{h+1}=\left\{\left(a_{0}, \cdots, a_{h}\right)\right\}$ is the parameter space of this family. We define $A$ to be the open set of $\mathbb{C}^{h+1}=\left\{\left(a_{0}, \cdots, a_{h}\right)\right\}$ that parametrizes the smooth hypersurfaces

$$
\left\{x \in \mathbf{P}^{n}: F\left(a_{0}, \cdots, a_{h}, x\right)=0\right\} .
$$

Theorem 1.2. If in the diagram (1.1)

$$
\phi \quad \text { is onto } \psi\left(T_{\left[f_{0}\right]} A\right),
$$

for a specific set of sections $L_{i}$ above, i.e. $C_{0}$ deforms with the hypersurface $X_{0}$ in all directions of $T_{\left[f_{0}\right]} A$ to the first order, then the twisted normal bundle

$$
N_{C_{0} / X_{0}}(1),
$$

is generated by global sections. In particular, if $X_{0}$ is a generic hypersurface and contains a smooth rational curve $C_{0}$,

$$
N_{C_{0} / X_{0}}(1)
$$

is generated by global sections.

In applying the theorem, we should note assumption (1.3) only requires ONE specific family $A$. An immediate corollary is 
Corollary 1.3. Assume $X_{0}$ is a smooth quintic threefold in $\mathbf{P}^{4}$, and assumption (1.3) in theorem 1.2 holds for some $A$ defined in definition 1.1. Let $d=\operatorname{deg}\left(\left.\mathcal{O}_{\mathbf{P}^{n}}(1)\right|_{C_{0}}\right)$. Then the splitting of the normal bundle $N_{C_{0} / X_{0}}$ must be

$$
N_{C_{0} / X_{0}} \simeq \mathcal{O}_{\mathbf{P}^{1}}(k) \oplus O_{\mathbf{P}^{1}}(-2-k)
$$

such that

$$
-1 \leq k \leq d-2 .
$$

In particular, (1.5) and (1.6) hold for a quintic 3-fold $X_{0}$ that is generic and contains a smooth rational curve $C_{0}$.

Proof. It is well-known that vector bundles over $\mathbf{P}^{1}$ can be decomposed as a direct sum of line bundles. Thus $N_{C_{0} / X_{0}}$ must be

$$
N_{C_{0} / X_{0}} \simeq \mathcal{O}_{\mathbf{P}^{1}}(k) \oplus O_{\mathbf{P}^{1}}(-2-k)
$$

where $k \geq-1$. Apply theorem 1.2 to obtain that

$$
N_{C_{0} / X_{0}}(1) \simeq \mathcal{O}_{\mathbf{P}^{1}}(d+k) \oplus O_{\mathbf{P}^{1}}(d-2-k)
$$

is generated by global sections. Thus

$$
d-2-k \geq 0 .
$$

This is the inequality in the corollary. In particular if $X_{0}$ is a quintic 3-fold that is generic and contains a smooth rational curve $C_{0}$, assumption (1.3) follows from lemma 2.2 below.

REMARK. Assumption (1.3) stresses the importance of the first order of deformations of the pair. Most of previous results, such as those listed above, have the different assumption: $X_{0}$ is generic, which is strictly stronger than assumption (1.3). This classical genericity assumption can be explained in the following. Let $M_{d}$ be the parameter space of embeddings $\mathbf{P}^{1} \rightarrow \mathbf{P}^{n}$, whose image has degree $d$. So $M_{d}$ is an open set of

$$
\mathbf{P}\left(\oplus_{n+1} \mathcal{O}_{\mathbf{P}^{n}}(d)\right) .
$$

The map $c_{0}$ represents a point in $M_{d}$ which is still denoted by $c_{0}$. Let

$$
\begin{aligned}
& \Gamma \subset M_{d} \times \mathbf{P}\left(H^{0}\left(\mathcal{O}_{\mathbf{P}^{n}}(h)\right)\right) \\
& \Gamma=\left\{([c],[f]): c^{*}(f)=0\right\} .
\end{aligned}
$$

Then the assumption: $X_{0}$ is generic is equivalent to the assumption that there is an irreducible component of $\Gamma$ containing $\left(c_{0},\left[f_{0}\right]\right)$, which dominates

$$
\mathbf{P}\left(H^{0}\left(\mathcal{O}_{\mathbf{P}^{n}}(h)\right)\right) .
$$

Our assumption (1.3) in this setting is equivalent to say that the Zariski tangent space $T_{\left(c_{0},\left[f_{0}\right]\right)} \Gamma$ is projected onto $T_{\left[f_{0}\right]} A$. It is not difficult to see the former assumption implies the latter one. We'll prove this assertion in section 2 .

Example 5.2 in section 5 indicates assumption (1.3) is strictly weaker than the assumption: $X_{0}$ is generic. 
The rest of the paper is organized as follows. In section 2, we give another description of first order deformation condition (1.3), that shows that it is weaker than the assumption: $X_{0}$ is generic. In section 3 , we study a family of smooth hypersurfaces. This is the main technique for the paper. In section 4 , we show that the first order assumption (1.3) leads to the positivity of the twisted normal bundle $N_{C_{0} / X_{0}}(1)$. This proves theorem 1.2 . In section 5 , we apply the result of theorem 1.2 to recover a classical result by Clemens, and give examples concerning our weaker assumption (1.3).

Acknowledgments. We would like to thank H. Clemens for the help and guidance, especially for his enlightening communication on theorems in sections $3,4([2])$.

2. First order deformations of the pair. In this section, we give another description of assumption (1.3), which will be used throughout. This is the description of the same map $\phi$ without factoring out isomorphism.

Let

$$
S \subset \mathbf{P}\left(H^{0}\left(\mathcal{O}_{\mathbf{P}^{n}}(h)\right)\right)
$$

be an irreducible subvariety that contains $\left[f_{0}\right]$ and is smooth at $\left[f_{0}\right]$. Let

$$
\begin{aligned}
& \mathcal{X}_{S} \subset \mathbf{P}^{n} \times S, \\
& \mathcal{X}_{S}=\{(x,[f]):[f] \in S, f(x)=0\} .
\end{aligned}
$$

be the universal hypersurface for $S \subset \mathbf{P}\left(H^{0}\left(\mathcal{O}_{\mathbf{P}^{n}}(h)\right)\right)$.

Let

$$
\begin{gathered}
\bar{c}_{0}: \mathbf{P}^{1} \rightarrow C_{0} \times\left\{\left[f_{0}\right]\right\} \subset \mathcal{X}_{S} \\
t \rightarrow\left(c_{0}(t),\left[f_{0}\right]\right)
\end{gathered}
$$

be the embedding determined by above embedding $c_{0}$. The projection

$$
P_{S}: \mathcal{X}_{S} \rightarrow S
$$

has a differential map

$$
T_{\left(q,\left[f_{0}\right]\right)} \mathcal{X}_{S} \rightarrow T_{\left[f_{0}\right]} S, \quad q \in C_{0}
$$

which can be extended to a bundle map

$$
\left(P_{S}\right)_{*}: \bar{c}_{0}^{*}\left(T_{\mathcal{X}_{S}}\right) \rightarrow T_{\left[f_{0}\right]} S \otimes \mathcal{O}_{\mathbf{P}^{1}}
$$

At last we obtain a morphism on the vector spaces

$$
P_{S}^{s}: H^{0}\left(\bar{c}_{0}^{*}\left(T_{\mathcal{X}_{S}}\right)\right) \rightarrow T_{\left[f_{0}\right]} S,
$$

where $T_{\left[f_{0}\right]} S \simeq H^{0}\left(T_{\left[f_{0}\right]} S \otimes \mathcal{O}_{\mathbf{P}^{1}}\right)$ is the space of global sections of the trivial bundle whose each fibre is $T_{\left[f_{0}\right]} S$.

LEMMA 2.1.

$$
\psi\left(T_{\left[f_{0}\right]} S\right) \subset \operatorname{image}(\phi)
$$

if and only if $P_{S}^{s}$ is surjective. 
Proof. Recall that $M_{d}$ is the parameter space of embeddings $\mathbf{P}^{1} \rightarrow \mathbf{P}^{n}$, whose image has degree $d$. Let $\mathcal{X}_{n}$ be the universal hypersurface for $S=\mathbf{P}\left(H^{0}\left(\mathcal{O}_{\mathbf{P}^{n}}(h)\right)\right)$ (defined in formula (2.2)). Recall

$$
\begin{aligned}
& \Gamma \subset M_{d} \times \mathbf{P}\left(H^{0}\left(\mathcal{O}_{\mathbf{P}^{n}}(h)\right)\right) \\
& \Gamma=\left\{(c,[f]): c^{*}(f)=0\right\} .
\end{aligned}
$$

be the incidence scheme containing the point $\left(c_{0},\left[f_{0}\right]\right)$. Let $T_{\left(c_{0},\left[f_{0}\right]\right)} \Gamma$ be the Zariski tangent space of $\Gamma$. Let $e$ be the evaluation map

$$
\begin{array}{ll}
e: \quad \Gamma \times \mathbf{P}^{1} \rightarrow \mathcal{X}_{n} \\
\\
(c,[f], t) \rightarrow(c(t),[f]) .
\end{array}
$$

Its differential map induces a bundle map

$$
e_{*}: \quad T_{\left(c_{0},\left[f_{0}\right]\right)} \Gamma \otimes \mathcal{O}_{\mathbf{P}^{1}} \rightarrow c_{0}^{*}\left(T_{\mathcal{X}_{n}}\right)
$$

It further induces a homomorphism on the cohomology groups:

$$
e^{s}: \quad T_{\left(c_{0},\left[f_{0}\right]\right)} \Gamma \rightarrow H^{0}\left(c_{0}^{*}\left(T_{\mathcal{X}_{n}}\right)\right),
$$

where $T_{\left(c_{0},\left[f_{0}\right]\right)} \Gamma=H^{0}\left(T_{\left(c_{0},\left[f_{0}\right]\right)} \Gamma \otimes \mathcal{O}_{\mathbf{P}^{1}}\right)$. Also there is a surjective map $\eta$ :

$$
T_{\left(C_{0},\left[f_{0}\right]\right)} \Gamma \quad \rightarrow \quad \mathbb{H}^{1}\left(T_{X_{0}} \rightarrow N_{C_{0} / X_{0}}\right),
$$

such that the following diagram commutes

$$
\begin{array}{ccccc}
T_{\left(C_{0},\left[f_{0}\right]\right)} \Gamma & = & T_{\left(C_{0},\left[f_{0}\right]\right)} \Gamma & \stackrel{\eta}{\downarrow} & \mathbb{H}^{1}\left(T_{X_{0}} \rightarrow N_{C_{0} / X_{0}}\right) \\
\downarrow^{s} & & & & \downarrow \phi \\
H^{0}\left(\bar{c}_{0}^{*}\left(T_{\mathcal{X}_{n}}\right)\right) & \stackrel{P_{n}^{s}}{\rightarrow} & T_{\left[f_{0}\right]} \mathbf{P}\left(H^{0}\left(\mathcal{O}_{\mathbf{P}^{n}}(h)\right)\right) & \stackrel{\psi}{\rightarrow} & H^{1}\left(T_{X_{0}}\right),
\end{array}
$$

where $P_{n}^{s}$ is the corresponding map in formula (2.3). Because

$$
T_{c_{0}} M_{d} \rightarrow H^{0}\left(c_{0}^{*}\left(T_{\mathbf{P}^{n}}\right)\right)
$$

is surjective (it is an isomorphism), $e^{s}$ has to be surjective. Then the lemma is true for $S=\mathbf{P}\left(H^{0}\left(\mathcal{O}_{\mathbf{P}^{n}}(h)\right)\right)$. Now we consider the subvariety $S \subset \mathbf{P}\left(H^{0}\left(\mathcal{O}_{\mathbf{P}^{n}}(h)\right)\right)$ in the lemma. If $\psi\left(T_{\left[f_{0}\right]} S\right) \subset$ image $(\phi)$, for any $\alpha \in T_{\left[f_{0}\right]} S$, we apply the diagram to find a section $\sigma \in H^{0}\left(\bar{c}_{0}^{*}\left(T_{\mathcal{X}_{n}}\right)\right)$ such that $P_{n}^{s}(\sigma)=\alpha$. Because $P_{n}^{s}(\sigma)=\alpha \in T_{\left[f_{0}\right]} S, \sigma$ must be in the subspace $H^{0}\left(\bar{c}_{0}^{*}\left(T_{\mathcal{X}_{S}}\right)\right)$ of $H^{0}\left(\bar{c}_{0}^{*}\left(T_{\mathcal{X}_{n}}\right)\right)$. Thus $P_{S}^{s}$ is surjective. Conversely we suppose $P_{S}^{s}$ is surjective. For any $\alpha \in T_{\left[f_{0}\right]} S$, using the commutative diagram, we obtain

$$
\psi(\alpha) \in \phi \circ \eta \circ\left(e^{s}\right)^{-1} \circ\left(P_{S}^{s}\right)^{-1}(\alpha) .
$$

We complete the proof.

LemMa 2.2. If $X_{0}$ is generic and contains a smooth rational curve $C_{0}$, or equivalently there is an irreducible component $\Gamma_{0}$ of the incidence scheme

$$
\left\{(c,[f]) \in M_{d} \times \mathbf{P}\left(H^{0}\left(\mathcal{O}_{\mathbf{P}^{n}}(h)\right)\right): c^{*}(f)=0\right\},
$$

such that $\Gamma_{0}$ dominates $\mathbf{P}\left(H^{0}\left(\mathcal{O}_{\mathbf{P}^{n}}(h)\right)\right)$ and $\left(c_{0},\left[f_{0}\right]\right) \in \Gamma_{0}$ is generic, then $\phi$ is surjective. 
Proof. In this proof, we consider the entire space of hypersurfaces, i.e.

$$
S=\mathbf{P}\left(H^{0}\left(\mathcal{O}_{\mathbf{P}^{n}}(h)\right)\right) .
$$

As before $\mathcal{X}_{n}$ denotes the universal hypersurface corresponding to $\mathbf{P}\left(H^{0}\left(\mathcal{O}_{\mathbf{P}^{n}}(h)\right)\right)$.

Let $c_{0}$ be as above and

$$
\bar{c}_{0}: \mathbf{P}^{1} \rightarrow X_{0} \times\left\{\left[f_{0}\right]\right\} \subset \mathcal{X}_{n}
$$

be the morphism that lifts the image $C_{0}$ to $\mathcal{X}_{n}$. The projection

$$
P: \mathcal{X}_{n} \rightarrow S
$$

induces a map on the sections of bundles over $\mathbf{P}^{1}$

$$
P^{s}: H^{0}\left(\bar{c}_{0}^{*}\left(T_{\mathcal{X}_{n}}\right)\right) \rightarrow T_{\left[f_{0}\right]} S,
$$

where $T_{\left[f_{0}\right]} S \simeq H^{0}\left(T_{\left[f_{0}\right]} S \otimes \mathcal{O}_{\mathbf{P}^{1}}\right)$ is the space of global sections of the trivial bundle whose each fibre is $T_{\left[f_{0}\right]} S$. Observe the commutative diagram

$$
\begin{aligned}
& T_{\left(c_{0},\left[f_{0}\right]\right)} \Gamma \stackrel{\left(e_{\Gamma}\right) *}{\rightarrow} \quad H^{0}\left(\bar{c}_{0}^{*}\left(T_{\mathcal{X}_{n}}\right)\right) \\
& \downarrow\left(\pi_{\Gamma}\right)_{*} \quad \downarrow P_{n}^{s} \\
& T_{\left[f_{0}\right]} S=T_{\left[f_{0}\right]} S \text {. }
\end{aligned}
$$

(see $(2.5)$ for $\left.P_{n}^{s}\right)$ where $\left(e_{\Gamma}\right)_{*}$ is induced from the differential of the evaluation $e_{\Gamma}$ :

$$
\begin{array}{ccc}
e_{\Gamma}: \Gamma \times \mathbf{P}^{1} & \rightarrow & \mathcal{X}_{n} \\
(c,[f], t) & \rightarrow & c(t) \times\{[f]\} .
\end{array}
$$

Since $f_{0}$ is generic and $\pi_{\Gamma}$ is dominant (by the assumption of the lemma), then $\left(c_{0},\left[f_{0}\right]\right) \in \Gamma$ is a generic point in $\Gamma_{0}$. Then the dominance of $\pi_{\Gamma}$ implies the surjectivity of $\left(\pi_{\Gamma}\right)_{*}$. Thus $P_{n}^{s}$ is surjective. By lemma 2.1, we proved lemma 2.2.

3. Deformation of hypersurfaces. In this section we do not assume that there is a rational curve $C_{0}$ as in section 1 . So we continue with the notations in the section 1 , however we do not assume the condition (1.2) because there is no rational curve $C_{0}$ in this section. Instead we assume

$$
\operatorname{div}\left(L_{i}\right) \neq \operatorname{div}\left(L_{j}\right), i \neq j .
$$

Recall that

$$
F\left(a_{1}, \cdots, a_{h}, x\right)=f_{0}(x)+\sum_{i=0}^{h} a_{i} L_{0}(x) \cdots \hat{L}_{i}(x) \cdots L_{h}(x), \quad\left(\text { omit } L_{i}\right)
$$

is the universal polynomial. Thus

$$
\{F=0\}=\mathcal{X}_{A} \subset \mathbf{P}^{n} \times A \text {. }
$$

is the universal hypersurface, which is smooth. Let $W \subset \mathbf{P}^{n}$ denote the complement of the proper subvariety

$$
\cup_{h \geq j>i \geq 0}\left\{L_{i}=L_{j}=0\right\}
$$


Let

$$
\mathcal{X}_{W}=\mathcal{X}_{A} \cap(W \times A) .
$$

Let

$$
u_{i}=L_{0} \frac{\partial}{\partial a_{0}}-L_{i} \frac{\partial}{\partial a_{i}}, i=1, \cdots, h
$$

be sections of $\mathcal{O}_{\mathbf{P}^{n}}(1) \otimes T_{A}$. Since $u_{i}$ annihilate $F$, they are tangent to $\mathcal{X}_{W}$. So let

$$
G(1) \subset T_{\mathcal{X}_{W}}(1)
$$

be the vector bundle of rank $h$ over $\mathcal{X}_{W}$ that is generated by the sections $u_{i}$.

We then have

\section{THEOREM 3.1.}

$$
\left.\frac{T_{\mathcal{X}_{W}}(1)}{G(1)} \simeq T_{(W \times A) / A}(1)\right|_{\mathcal{X}_{W}}
$$

where $T_{(W \times A) / A}(1)=\left(T_{W}(1) \oplus\{0\}\right)$ is the twisted relative tangent bundle of the projection $W \times A \rightarrow A$.

REMARK. This theorem does not require additional assumptions. This is a fact about this special type of family of hypersurfaces.

Proof. Consider the exact sequence

$$
0 \rightarrow \frac{T_{\mathcal{X}_{W}}(1)}{G(1)} \rightarrow \frac{T_{(W \times A)}(1)}{G(1)} \rightarrow \mathcal{D} \rightarrow 0
$$

of bundles over $\mathcal{X}_{W}$, where $\mathcal{D}$ is some quotient bundle over $\mathcal{X}_{W}$. It is easy to see that

$$
c_{1}(\mathcal{D})=\left.c_{1}\left(\mathcal{O}_{\mathbf{P}^{n}}(h+1)\right)\right|_{\mathcal{X}_{W}}
$$

Let $s$ be a generic section of $\mathcal{O}_{\mathbf{P}^{n}}(1)$. Let $\sigma$ be the reduction of $s \frac{\partial}{\partial a_{0}}$ in $\frac{T_{(W \times A)}(1)}{G(1)}$. Notice that the zero-locus of $\sigma$ is given by

$$
\operatorname{div}(\sigma)=\operatorname{div}\left(s L_{1} \cdots L_{h}\right) .
$$

Since $s L_{1} \cdots L_{h} \in H^{0}\left(\mathcal{O}_{\mathbf{P}^{n}}(h+1)\right), \sigma$ splits the sequence $(3.5)$. If $L_{s} \subset \frac{T_{(W \times A)}(1)}{G(1)}$ is the line bundle generated by $\sigma$,

$$
L_{s} \oplus \frac{T_{\mathcal{X}_{W}}(1)}{G(1)}=\frac{T_{(W \times A)}(1)}{G(1)},
$$

as bundles over $\mathcal{X}_{W}$. Secondly, we have another exact sequence

$$
0 \rightarrow T_{(W \times A) / A}(1) \rightarrow \frac{T_{(W \times A)}(1)}{G(1)} \rightarrow \mathcal{D}^{\prime} \rightarrow 0 .
$$

of bundles over $\mathcal{X}_{W}$, where $\mathcal{D}^{\prime}$ is some quotient bundle over $\mathcal{X}_{W}$. By direct computation (note $G(1)$ is a trivial bundle), we obtain:

$$
c_{1}\left(\mathcal{D}^{\prime}\right)=c_{1}\left(T_{W \times A / W}(1)\right)=\left.(h+1)\left(c_{1}\left(\mathcal{O}_{\mathbf{P}^{n}}(1)\right)\right)\right|_{\mathcal{X}_{W}} .
$$


As above, $\sigma$ splits this sequence (3.9). Hence

$$
L_{s} \oplus T_{(W \times A) / A}(1)=\frac{T_{(W \times A)}(1)}{G(1)} .
$$

Comparing (3.8), (3.10), we obtain

$$
\frac{T_{\mathcal{X}_{W}}(1)}{G(1)} \simeq T_{(W \times A) / A}(1),
$$

over $\mathcal{X}_{W}$.

4. Positivity of the twisted normal bundle. In this section we prove theorem 1.2. We continue with the notations in section 1 . In particular $C_{0}$ is a smooth rational curve in $X_{0}$.

Proof of Theorem 1.2. Denote $C_{0} \times\left\{\left[f_{0}\right]\right\}$ by $\bar{C}_{0}$. Because of our assumption (1.2), $C_{0}$ completely lies in $W$. Thus we have the exact sequence of bundles

$$
0 \rightarrow \bar{c}_{0}^{*}\left(T_{\bar{C}_{0}}(1)\right) \rightarrow \bar{c}_{0}^{*}\left(\frac{T_{\mathcal{X}_{A}}(1)}{G(1)}\right) \rightarrow \bar{c}_{0}^{*}\left(\frac{\left.T_{\mathcal{X}_{A}}(1)\right)}{G(1)+T_{\bar{C}_{0}}(1)}\right) \rightarrow 0
$$

By theorem 3.1, we have two exact sequences,

$$
\begin{aligned}
& 0 \rightarrow \bar{c}_{0}^{*}\left(T_{\bar{C}_{0}}(1)\right) \rightarrow \bar{c}_{0}^{*}\left(\frac{T_{\mathcal{X}_{A}}(1)}{G(1)}\right) \quad \rightarrow \quad \bar{c}_{0}^{*}\left(\frac{\left.T_{\mathcal{X}_{A}}(1)\right)}{G(1)+T_{\bar{C}_{0}}(1)}\right) \rightarrow 0 \\
& 0 \rightarrow \bar{c}_{0}^{*}\left(T_{\bar{C}_{0}}(1)\right) \rightarrow \bar{c}_{0}^{*}\left(T_{\left(\mathbf{P}^{n} \times A\right) / A}(1)\right) \rightarrow c_{0}^{*}\left(N_{C_{0} / \mathbf{P}^{n}}(1)\right) \rightarrow 0 .
\end{aligned}
$$

where $I$ is the isomorphism in theorem 3.1. Notice in theorem 3.1, the isomorphism $I$ is restricted to the identity map on $\bar{c}_{0}^{*}\left(T_{\bar{C}_{0}}(1)\right)$. Notice that the first half of the diagram (4.2),

$$
\begin{aligned}
& 0 \rightarrow \bar{c}_{0}^{*}\left(T_{\bar{C}_{0}}(1)\right) \rightarrow \bar{c}_{0}^{*}\left(\frac{T_{\mathcal{X}_{A}}(1)}{G(1)}\right) \\
& 0 \rightarrow \bar{c}_{0}^{*}\left(T_{\bar{C}_{0}}(1)\right) \rightarrow \bar{c}_{0}^{*}\left(T_{\left(\mathbf{P}^{n} \times A\right) / A}(1)\right) .
\end{aligned}
$$

is commutative. So we obtain

$$
c_{0}^{*}\left(N_{C_{0} / \mathbf{P}^{n}}(1)\right) \simeq \bar{c}_{0}^{*}\left(\frac{\left.T_{\mathcal{X}_{A}}(1)\right)}{G(1)+T_{\bar{C}_{0}}(1)}\right) .
$$

This isomorphism gives us another exact sequence

$$
0 \rightarrow \bar{c}_{0}^{*}(G(1)) \rightarrow \bar{c}_{0}^{*}\left(\frac{T_{\mathcal{X}_{A}}(1)}{T_{\bar{C}_{0}}(1)}\right) \rightarrow c_{0}^{*}\left(N_{C_{0} / \mathbf{P}^{n}}(1)\right) \rightarrow 0 .
$$

To see the positivity of bundles, we observe that since $H^{1}\left(c_{0}^{*} G(1)\right)=0\left(c_{0}^{*} G(1)\right.$ is a trivial bundle over $\left.\mathbf{P}^{1}\right)$, sections of $c_{0}^{*}\left(N_{C_{0} / \mathbf{P}^{n}}(1)\right)$ can be lifted to sections of

$$
\bar{c}_{0}^{*}\left(\frac{T_{\mathcal{X}_{A}}(1)}{T_{\bar{C}_{0}}(1)}\right) .
$$

Then $\bar{c}_{0}^{*}\left(\frac{T_{\mathcal{X}_{A}}(1)}{T_{\bar{C}_{0}}(1)}\right)$ must be generated by global sections because $c_{0}^{*}\left(N_{C_{0} / \mathbf{P}^{n}}(1)\right)$ and $c_{0}^{*} G(1)$ are. The proof is now completed with the last observation that $\bar{c}_{0}^{*}\left(\frac{T_{\mathcal{X}_{A}}(1)}{T_{\bar{C}_{0}}(1)}\right)$ is 
mapped onto $N_{C_{0} / X_{0}}(1)$. This is the only place where assumption (1.3) is used. The following is the argument for this surjection.

Because $\phi$ is onto $\psi(A), P_{A}^{s}$ is onto $T_{f_{0}} A$. This gives us a natural bundle decomposition of $\bar{c}_{0}^{*}\left(T_{\mathcal{X}_{A}}(1)\right)$ in the following way: Note $\bar{c}_{0}^{*}\left(T_{\mathcal{X}_{A}}(1)\right)$ has sections $\left(P_{A}^{s}\right)^{-1}\left(\frac{\partial}{\partial a_{j}}\right), j=0, \cdots, h$. Let $\sigma_{i} \in\left(P_{A}^{s}\right)^{-1}\left(\frac{\partial}{\partial a_{j}}\right), i=0, \cdots, h$ be a vector in each inverse $\left(P_{A}^{s}\right)^{-1}\left(\frac{\partial}{\partial a_{j}}\right)$. Then all sections $\left\{\sigma_{j}\right\}_{j}$ generate a trivial subbundle $\mathcal{E}$

$$
\mathcal{E}=\oplus_{h+1} \mathcal{O}_{\mathbf{P}^{1}}
$$

This subbundle gives a decomposition

$$
\bar{c}_{0}^{*}\left(T_{\mathcal{X}_{A}}\right) \simeq \mathcal{E} \oplus \bar{c}_{0}^{*}\left(T_{\mathcal{X}_{A} / A}\right),
$$

Tensoring it with $\bar{c}_{0}^{*}\left(\mathcal{O}_{\mathbf{P}^{n}}(1)\right)$, we obtain

$$
\bar{c}_{0}^{*}\left(T_{\mathcal{X}_{A}}(1)\right) \simeq \mathcal{E}(1) \oplus \bar{c}_{0}^{*}\left(T_{\mathcal{X}_{A} / A}(1)\right) .
$$

Notice

$$
\frac{\bar{c}_{0}^{*}\left(T_{\mathcal{X}_{A}}(1)\right)}{\mathcal{E}(1) \oplus \bar{c}_{0}^{*}\left(T_{\bar{C}_{0}}(1)\right)} \simeq c_{0}^{*}\left(N_{C_{0} / X_{0}}(1)\right) .
$$

Thus there is an exact sequence

$$
\bar{c}_{0}^{*}\left(\frac{T_{\mathcal{X}_{A}}(1)}{T_{\bar{C}_{0}}(1)}\right) \rightarrow c_{0}^{*}\left(N_{C_{0} / X_{0}}(1)\right) \rightarrow 0 .
$$

Because $\bar{c}_{0}^{*}\left(\frac{T_{\mathcal{X}_{A}}(1)}{T_{\bar{C}_{0}}(1)}\right)$ is generated by global sections, then so is $c_{0}^{*}\left(N_{C_{0} / X_{0}}(1)\right)$. This completes the proof.

\section{Application.}

Corollary 5.1. Assume $X_{0}$ is a smooth hypersurface in $\mathbf{P}^{n}$ of degree $h$. Also assume assumption (1.3) in theorem 1.2 holds.

Then

$$
h \leq 2 n-2 .
$$

Proof. By the isomorphism in theorem 3.1, we have the exact sequence

$$
0 \rightarrow \bar{c}_{0}^{*}(G(1)) \quad \rightarrow \quad \bar{c}_{0}^{*}\left(T_{\mathcal{X}_{A}}(1)\right) \quad \rightarrow \quad c_{0}^{*}\left(T_{\mathbf{P}^{n}}(1)\right) \quad \rightarrow \quad 0 .
$$

As before

$$
H^{1}\left(\bar{c}_{0}^{*} G(1)\right)=0
$$

Hence

$$
0 \rightarrow H^{0}\left(\bar{c}_{0}^{*} G(1)\right) \rightarrow H^{0}\left(\bar{c}_{0}^{*}\left(T_{\mathcal{X}_{A}}(1)\right)\right) \rightarrow H^{0}\left(c_{0}^{*}\left(T_{\mathbf{P}^{n}}(1)\right)\right) \rightarrow 0
$$

Therefore

$$
h^{0}\left(\bar{c}_{0}^{*}\left(T_{\mathcal{X}_{A}}(1)\right)\right)=h^{0}\left(c_{0}^{*}\left(T_{\mathbf{P}^{n}}(1)\right)\right)+h .
$$


To calculate $H^{0}\left(c_{0}^{*}\left(T_{\mathbf{P}^{n}}(1)\right)\right)$, we consider the twisted Euler sequence

$$
0 \rightarrow c_{0}^{*}\left(\mathcal{O}_{\mathbf{P}^{n}}(1)\right) \rightarrow c_{0}^{*}\left(\oplus_{n+1} \mathcal{O}_{\mathbf{P}^{n}}(2)\right) \rightarrow c_{0}^{*}\left(T_{\mathbf{P}^{n}}(1)\right) \rightarrow 0 .
$$

Because $H^{1}\left(c_{0}^{*}\left(\mathcal{O}_{\mathbf{P}^{n}}(1)\right)\right)=H^{0}\left(\mathcal{O}_{\mathbf{P}^{1}}(-d-2)\right)=0$, we obtain

$$
0 \rightarrow H^{0}\left(c_{0}^{*}\left(\mathcal{O}_{\mathbf{P}^{n}}(1)\right)\right) \rightarrow H^{0}\left(c_{0}^{*}\left(\oplus_{n+1} \mathcal{O}_{\mathbf{P}^{n}}(2)\right)\right) \rightarrow H^{0}\left(c_{0}^{*}\left(T_{\mathbf{P}^{n}}(1)\right)\right) \rightarrow 0 .
$$

Hence we find

$$
\begin{aligned}
h^{0}\left(c_{0}^{*}\left(T_{\mathbf{P}^{n}}(1)\right)\right) & =h^{0}\left(c_{0}^{*}\left(\oplus_{n+1} \mathcal{O}_{\mathbf{P}^{n}}(2)\right)\right)-h^{0}\left(c_{0}^{*}\left(\mathcal{O}_{\mathbf{P}^{n}}(1)\right)\right) \\
& =(2 d+1)(n+1)-(d+1) .
\end{aligned}
$$

To calculate $H^{0}\left(\bar{c}_{0}^{*}\left(T_{\mathcal{X}_{A}}(1)\right)\right)$, we note that $\phi$ is onto $\psi\left(T_{\left[f_{0}\right]} A\right)$. Then we obtain the decomposition (4.6):

$$
\bar{c}_{0}^{*}\left(T_{\mathcal{X}_{A}}(1)\right)=\mathcal{E}(1) \oplus c_{0}^{*}\left(T_{X_{0}}(1)\right) .
$$

where

$$
\mathcal{E}(1) \simeq \oplus_{h+1} c_{0}^{*}\left(\mathcal{O}_{\mathbf{P}^{n}}(1)\right) \simeq \oplus_{h+1} \mathcal{O}_{\mathbf{P}^{1}}(d)
$$

Hence

$$
h^{0}\left(\bar{c}_{0}^{*}\left(T_{\mathcal{X}_{A}}(1)\right)\right)=(h+1)(d+1)+h^{0}\left(c_{0}^{*}\left(T_{X_{0}}(1)\right)\right) .
$$

Combining formulas (5.3), (5.5) and (5.7), we obtain that

$$
h^{0}\left(c_{0}^{*}\left(T_{X_{0}}(1)\right)\right)+(h-2 n) d-(n-1)=0 .
$$

Since

$$
\begin{aligned}
& h^{0}\left(c_{0}^{*}\left(T_{X_{0}}(1)\right)\right)=h^{0}\left(N_{C_{0} / X_{0}}(1)\right)+h^{0}\left(T_{\mathbf{P}^{1}} \otimes c_{0}^{*}\left(\mathcal{O}_{\mathbf{P}^{n}}(1)\right)\right) \\
& =h^{0}\left(N_{C_{0} / X_{0}}(1)\right)+d+3,
\end{aligned}
$$

formula (5.8) becomes

$$
(h-2 n+1) d+h^{0}\left(N_{C_{0} / X_{0}}(1)\right)-(n-4)=0 .
$$

To show $h \leq 2 n-2$, it suffices to prove that

$$
h^{0}\left(N_{C_{0} / X_{0}}(1)\right)-(n-4)>0 .
$$

Applying theorem 1.2, we obtain that

$$
h^{0}\left(N_{C_{0} / X_{0}}(1)\right) \geq \operatorname{Rank}\left(N_{C_{0} / X_{0}}(1)\right)=n-2 .
$$

This completes the proof.

REMARK. There are two previously well-known results that are related to corollary 5.1:

(1). H. Clemens proved a theorem in [3], that implies if $X_{0}$ is a generic hypersurface containing an immersed rational curve $C_{0}$, then

$$
\operatorname{deg}\left(X_{0}\right) \leq 2 n-2 .
$$


The result later was improved by C. Voisin [9], [10]:

(2) If $X_{0}$ is a generic hypersurface containing $C_{0}$ which is any rational curve, then

$$
\operatorname{deg}\left(X_{0}\right) \leq 2 n-3, \quad \text { for } n \geq 4
$$

and the equality holds for a line in a generic hypersurface of degree $2 n-3$.

Both authors in their papers addressed more general situations.

Our theorem 1.2 is valid for immersed rational curves on hypersurfaces. Then using lemma 2.2 and corollary 5.1, we recover H. Clemens' result mentioned in (1). So corollary 5.1 implies Clemens' result (1), but they are not equivalent. Even though Clemens' bound in corollary 5.1 is worse than Voisin's, it is still sharp under our weaker assumption. Please see the following example 5.2 for this.

Example 5.2 is in the case where assumption (1.3) holds for a specific family $A$, but it does not hold for all such families $A$. Example 5.3 is in the case where assumption (1.3) does not hold for any family $A^{1}$.

ExAmple 5.2. This example constructs $X_{0}, C_{0}, A$ of theorem 1.2 satisfying assumption (1.3), and they further satisfy

(1) $\operatorname{deg}\left(X_{0}\right)=2 n-2$ ( This is the minimum degree of $X_{0}$ by corollary 5.1 ).

(2) $C_{0}$ does not deform to all hypersurfaces to the first order.

( assertion (2) will be proved elsewhere).

Let $x_{0}, \cdots, x_{n}$ be homogeneous coordinates for $\mathbf{P}^{n}$. The construction is based on the break-down of $\mathbf{P}^{n}$ to smaller subspaces. Let

$$
\mathbf{P}_{\text {sub }}^{1} \subset \mathbf{P}_{\text {sub }}^{2} \subset \mathbf{P}^{n}
$$

be subspaces defined as follows:

$$
\mathbf{P}_{\text {sub }}^{2}=\left\{x_{0}=\cdots=x_{n-3}=0\right\}, \mathbf{P}_{\text {sub }}^{1}=\mathbf{P}_{\text {sub }}^{2} \cap \mathbf{P}_{\text {sub }}^{n-1},
$$

where

$$
\mathbf{P}_{s u b}^{n-1}=\left\{x_{n}=0\right\}
$$

In the following, we always regard homogeneous polynomials on subspaces

$$
\mathbf{P}_{\text {sub }}^{i}, i=1,2, n-1
$$

as polynomials of the same degree on $\mathbf{P}^{n}$, i.e. we use the natural inclusion

$$
H^{0}\left(\mathcal{O}_{\mathbf{P}_{\text {sub }}^{i}}(r)\right) \subset H^{0}\left(\mathcal{O}_{\mathbf{P}^{n}}(r)\right), \text { for } i=1,2, n-1 .
$$

Next we construct three varieties: $X_{0}, C_{0}, A$ in theorem 1.2 .

(1) $X_{0}$ :

Let $g_{0}, \cdots, g_{n-3}$ be generic sections in $H^{0}\left(\mathcal{O}_{\mathbf{P}_{\text {sub }}^{n-1}}(2 n-3)\right)$.

Let

$$
q_{1}=\sum_{i=0}^{2} x_{n-i}^{2} \in H^{0}\left(\mathcal{O}_{\mathbf{P}_{\text {sub }}^{2}}(2)\right) .
$$

\footnotetext{
${ }^{1}$ It turns out that these two examples cover all situations regarding assumption (1.3), but excluding the assumption: $X_{0}$ is generic.
} 
Let $q_{2} \in H^{0}\left(\mathcal{O}_{\mathbf{P}^{n}}(2 n-4)\right)$ be a section

$$
q_{2}=x_{n}^{2(n-2)}+p
$$

where $p \in H^{0}\left(\mathcal{O}_{\mathbf{P}_{\text {sub }}^{n-1}}(2 n-4)\right)$ is generic.

Now we construct the smooth hypersurface $X_{0}=\operatorname{div}\left(f_{0}\right)$ of degree $2 n-2$ :

Let

$$
f_{0}=q_{1} q_{2}+\sum_{k=0}^{n-3} x_{k} g_{k} .
$$

Such $X_{0}=\operatorname{div}\left(f_{0}\right)$ is smooth (see Appendix).

(2) $C_{0}$ :

Let the rational curve $C_{0}$ be

$$
C_{0}=\left\{x_{0}=\cdots=x_{n-3}=q_{1}=0\right\} .
$$

Since $C_{0}$ is a smooth plane conic in $X_{0}$, it is a smooth rational curve. Let

$$
c_{0}: \mathbf{P}^{1} \rightarrow C_{0} \subset X_{0}
$$

be an isomorphism.

(3) $A$ :

Let $L_{0}, \cdots, L_{h}$ be generic $h+1$ sections in the pencil

$$
\operatorname{span}\left(x_{n-2}, x_{n-1}\right)=H^{0}\left(\mathcal{O}_{\mathbf{P}_{\text {sub }}^{1}}(1)\right) \subset H^{0}\left(\mathcal{O}_{\mathbf{P}^{n}}(1)\right),
$$

where $h=2 n-2$. Then the condition (1.2) is satisfied, i.e.,

$$
\left\{L_{i}=0\right\} \cap\left\{L_{j}=0\right\} \cap C_{0}=\emptyset, \quad i \neq j .
$$

Let $A \subset H^{0}\left(\mathcal{O}_{\mathbf{P}^{n}}(h)\right)$ be constructed with $L_{j}, j=0, \cdots, h$ as in definition 1.1.

For fixed generic $q_{2}, q_{3}, g_{k}$, corresponding maps $\psi$ and $\phi$ in theorem 1.2 satisfy

$$
\psi(A) \subset \operatorname{image}(\phi) .
$$

See Appendix for the proof of it. The example shows our bound $2 n-2$ is the sharp bound under assumption (1.3). Together with Voisin's result (2), this indicates assumption (1.3) is strictly weaker than the classical assumption: $X_{0}$ is generic.

EXAMPLE 5.3. Let's consider the lines in the Fermat quintic threefold. Let $x_{0}, \cdots, x_{4}$ be the homogeneous coordinates for $\mathbf{P}^{4}$. We consider $f_{0}$ to be the Fermat quintic

$$
x_{0}^{5}+x_{1}^{5}+\cdots+x_{4}^{5}
$$

Let $C_{0}$ be the line in $\mathbf{P}^{4}$ connecting two points

$$
[1,-1,0,0,0],\left[0,0, a_{1}, a_{2}, a_{3}\right]
$$


with $a_{1}^{5}+a_{2}^{5}+a_{3}^{5}=0$. Then $C_{0} \subset X_{0}=\operatorname{div}\left(f_{0}\right)$. We can find

$$
N_{C_{0}} X_{0} \simeq \mathcal{O}_{\mathbf{P}^{1}}(-1) \oplus \mathcal{O}_{\mathbf{P}^{1}}(3) .
$$

Corollary 1.3 says that if assumption (1.3) holds, then

$$
N_{C_{0}} X_{0} \simeq \mathcal{O}_{\mathbf{P}^{1}}(-1) \oplus \mathcal{O}_{\mathbf{P}^{1}}(-1) .
$$

(because $d=1)$. This contradiction says $\left(C_{0}, f_{0}\right)$ does not deform to all hypersurfaces in $A$ to the first order, i.e. assumption (1.3) does not hold. This result is stronger than Albano and Katz's result, Prop. 2.1, in [1], which says $\left(C_{0}, f_{0}\right)$ does not globally deform to all hypersurfaces.

Appendix. (1) $X_{0}$ is smooth. To see that, we specialize it at

$$
g_{k}=x_{k}^{2 n-3}, g_{0}=q_{3}+x_{0}^{2 n-3}, q_{2}=x_{n}^{2 n-4}
$$

where

$$
q_{3} \in H^{0}\left(\mathcal{O}_{\mathbf{P}^{1}}(2 n-3)\right)
$$

is generic. Then if $z \in X_{0}$ is a singular point, it must satisfy

$$
\left\{\begin{array}{cc}
x_{1}=\cdots=x_{n-3}=0, & \left.\left(\left.\frac{\partial f_{0}}{\partial x_{k}}\right|_{z}=0, k=1, \cdots, n-3\right)\right) \\
\left.\frac{\partial\left(q_{1} q_{2}+x_{0}\left(x_{0}^{2 n-3}+q_{3}\right)\right)}{\partial \alpha_{2 n-3}}\right|_{z}=0, & \left(\alpha \in T_{q} \mathbb{C}^{4}, \mathbb{C}^{4}=\left(x_{0}, x_{n}, x_{n-1}, x_{n-2}\right)\right) \\
\left.\left(q_{1} q_{2}+x_{0}\left(x_{0}^{2 n-3}+q_{3}\right)\right)\right|_{z}=0, & \left(f_{0}(z)=0\right) .
\end{array}\right.
$$

It is easy to see such point $z$ must be a zero of

$$
q_{1}=x_{n}=x_{0}=q_{3}=0, \quad \text { and } x_{0}=\cdots=x_{n-3}=0
$$

which does not exist for generic $q_{3}$. Hence $X_{0}$ is smooth in this case. Now moving $q_{2}, g_{k}$ to generic sections, we obtain that if all

$$
q_{2}, g_{k}, k=0, \cdots, n-3
$$

are generic, $X_{0}$ is a smooth hypersurface.

(2) Proof of (5.17). The general idea follows from the direct calculation. We compute image $(\phi)$ to be the set

$$
\left.\left(\sum_{i=0}^{2 n-2} z_{i} x_{n-1}^{i} x_{n-2}^{2 n-2-i}\right)\right|_{C_{0}}, \text { for all complex numbers } z_{i}
$$

and $\operatorname{image}\left(\nu^{s}\right)$ is the set

$$
\left.\left(\sum_{i=0}^{n} a_{i}\left(x_{0}, \cdots, x_{n}\right) \frac{\partial f_{0}}{\partial x_{i}}\right)\right|_{C_{0}}, \text { for all linear forms } a_{i}\left(x_{0}, \cdots, x_{n}\right) .
$$

Assumption (1.3) says the former set is contained in the latter. We would like to show the former set of polynomials in $x_{n-1}, x_{n-2}$ (not evaluated at $C_{0}$ ) is already 
contained in the latter set of polynomials (before evaluated at $C_{0}$ ). The following is the detailed computation.

First we'll build a diagram (5.21) below by the following maps: Let $\nu^{s}$ :

$$
\begin{aligned}
& H^{0}\left(c_{0}^{*}\left(T_{\mathbf{P}^{n}}\right)\right) \stackrel{\nu^{s}}{\rightarrow} \quad H^{0}\left(\mathcal{O}_{\mathbf{P}^{1}}(2 h)\right) \\
& {\left[\alpha_{0}, \cdots, \alpha_{n}\right] \rightarrow \sum_{i=0}^{n} \alpha_{i} c_{0}^{*}\left(\frac{\partial f_{0}}{\partial x_{i}}\right) .}
\end{aligned}
$$

where $\alpha_{i} \in H^{0}\left(\mathcal{O}_{\mathbf{P}^{1}}(2)\right)$ and $\left[\alpha_{0}, \cdots, \alpha_{n}\right]$ represents a vector in

$$
H^{0}\left(c_{0}^{*}\left(T_{\mathbf{P}^{n}}\right)\right) \simeq M_{2} .
$$

Let $\mu^{s}$ :

$$
\begin{array}{cl}
T_{\left[f_{\left.f_{1}\right]} A\right.} \stackrel{\mu^{s}}{\rightarrow} H^{0}\left(\mathcal{O}_{\mathbf{P}^{1}}(2 h)\right) \\
\frac{\partial}{\partial a} & \rightarrow-c_{0}^{*}\left(\frac{\partial F}{\partial a}\right),
\end{array}
$$

where $F$ is the universal polynomial of the family of hypersurfaces determined by $A$ (defined at the beginning of section 3). Let $P_{1}^{s}$ be the obvious projection map

$$
H^{0}\left(\bar{c}_{0}^{*}\left(T_{\mathcal{X}_{A}}\right)\right) \stackrel{P_{\rightarrow}^{s}}{\rightarrow} H^{0}\left(c_{0}^{*}\left(T_{\mathbf{P}^{n}}\right)\right) .
$$

Then all these maps fit into the commutative diagram

$$
\begin{array}{ccc}
H^{0}\left(\bar{c}_{0}^{*}\left(T_{\mathcal{X}_{A}}\right)\right) & \stackrel{P_{A}^{s}}{\rightarrow} & T_{\left[f_{0}\right]} A \\
\downarrow P_{1}^{s} & & \downarrow \mu^{s} \\
H^{0}\left(c_{0}^{*}\left(T_{\mathbf{P}^{n}}\right)\right) & \stackrel{\nu^{s}}{\rightarrow} & H^{0}\left(\mathcal{O}_{\mathbf{P}^{1}}(2 h)\right) .
\end{array}
$$

By theorem 2.1, we need to show the surjectivity of $P_{A}^{s}$. This commutative diagram (5.21) shows that it suffices to show

$$
\operatorname{image}\left(\mu^{s}\right) \subset \operatorname{image}\left(\nu^{s}\right) .
$$

Note

$$
A=H^{0}\left(\mathcal{O}_{\mathbf{P}_{\text {sub }}^{1}}(h)\right) \subset H^{0}\left(\mathcal{O}_{\mathbf{P}^{n}}(h)\right),
$$

where the inclusion is obtained by the natural extension of the sections of $\mathcal{O}_{\mathbf{P}_{\text {sub }}^{1}}(h)$ to $\mathcal{O}_{\mathbf{P}^{n}}(h)$ (as indicated before). By the definition of $\mu^{s}$,

$$
\mu^{s}\left(T_{\left[f_{0}\right]} A\right)=c_{0}^{*}\left(H^{0}\left(\mathcal{O}_{\mathbf{P}_{s u b}^{1}}(h)\right)\right) .
$$

Next we would like to see that the image of $\nu^{s}$ contains all polynomials in

$$
c_{0}^{*}\left(H^{0}\left(\mathcal{O}_{\mathbf{P}_{\text {sub }}^{1}}(h)\right)\right) .
$$

To see this, we'll build another diagram (5.27) below in the following way. First observe the natural inclusion:

$$
H^{0}\left(\mathcal{O}_{\mathbf{P}_{\text {sub }}^{1}}(1)\right) \subset H^{0}\left(\mathcal{O}_{\mathbf{P}^{n}}(1)\right) .
$$


Then notice

$$
H^{0}\left(\mathcal{O}_{\mathbf{P}_{\text {sub }}^{1}}(1)\right) \frac{\partial}{\partial x_{j}}, j=0, \cdots, n-1
$$

are 2 dimensional sub-spaces of global sections of the bundle $T_{\mathbf{P}^{n}}$. Let

$$
\mathcal{H}=\oplus_{j=0}^{n-1} H^{0}\left(\mathcal{O}_{\mathbf{P}_{\text {sub }}^{1}}(1)\right) \frac{\partial}{\partial x_{j}} \subset H^{0}\left(T_{\mathbf{P}^{n}}\right) .
$$

Then $\operatorname{dim}(\mathcal{H})=2 n$.

There is a homomorphism

$$
\begin{array}{ccc}
\mathcal{H} & \stackrel{\xi}{\rightarrow} & H^{0}\left(\mathcal{O}_{\mathbf{P}^{n}}(h)\right) \\
\sum_{j=0}^{n-1} l_{j}\left(x_{n-1}, x_{n-2}\right) \frac{\partial}{\partial x_{j}} & \rightarrow & \sum_{j=0}^{n-1} l_{j}\left(x_{n-1}, x_{n-2}\right) \frac{\partial f_{0}}{\partial x_{j}} .
\end{array}
$$

Let $\operatorname{Pr}$ be the morphism of linear spaces

$$
\oplus_{r=0}^{\infty} H^{0}\left(\mathcal{O}_{\mathbf{P}^{n}}(r)\right) \rightarrow \oplus_{r=0}^{\infty} H^{0}\left(\mathcal{O}_{\mathbf{P}_{\text {sub }}^{1}}(r)\right)
$$

( $P r$ will not be the natural pullback map). To describe $P r$, it suffices to define it on a basis. Let $\left(x_{n}\right)^{m} G$ be the monomials in $x_{0}, \cdots, x_{n}$ that form a basis for $H^{0}\left(\mathcal{O}_{\mathbf{P}^{n}}(r)\right)$ under the $x_{0}, \cdots, x_{n}$ coordinates, where $G \in H^{0}\left(\mathcal{O}_{\mathbf{P}_{\text {sub }}^{n-1}}(r-m)\right)$. Define

$$
\begin{gathered}
\operatorname{Pr}\left(\left(x_{n}\right)^{m} G\right)=0, \quad \text { if } m \text { is odd, } \\
\operatorname{Pr}\left(\left(x_{n}\right)^{m} G\right)=\left(-\left(x_{n-1}^{2}+x_{n-2}^{2}\right)\right)^{m / 2} \tilde{G}, \quad \text { if } m \text { is even, }
\end{gathered}
$$

where $\tilde{G}=\left.G\right|_{x_{0}=\cdots=x_{n-3}=0}$. These maps form a diagram

$$
\begin{array}{ccccc}
\mathcal{H} & \stackrel{\xi}{\rightarrow} & H^{0}\left(\mathcal{O}_{\mathbf{P}^{n}}(h)\right) & = & H^{0}\left(\mathcal{O}_{\mathbf{P}^{n}}(h)\right) \\
\downarrow c_{0}^{*} & & & & \downarrow P_{r r} \\
H^{0}\left(c_{0}^{*}\left(T_{\mathbf{P}^{n}}\right)\right) & \stackrel{\nu^{s}}{\rightarrow} & H^{0}\left(\mathcal{O}_{\mathbf{P}^{1}}(2 h)\right) & \stackrel{c_{0}^{*}}{\leftarrow} & H^{0}\left(\mathcal{O}_{\mathbf{P}_{\text {sub }}^{1}}(h)\right) .
\end{array}
$$

that is commutative because of the choices of $q_{2}$ and $g_{k}$ (No monomials of $x_{n}$-factor in $g_{k}$ and only a monomial of $x_{n}^{e v e n}$-factor in $\left.q_{2}\right){ }^{2}$

Our observation is that $\operatorname{Pr} \circ \xi$ is surjective, because the image of the composition map $\operatorname{Pr} \circ \xi$ is just

$$
\sum_{i=1}^{2} l_{n-i}\left(x_{n-1}, x_{n-2}\right) \operatorname{Pr}\left(\frac{\partial q_{1}}{\partial x_{n-i}} q_{2}\right)+\sum_{k=0}^{n-3} l_{k}\left(x_{n-1}, x_{n-2}\right) \operatorname{Pr}\left(g_{k}\right),
$$

(a polynomial in $x_{n-1}, x_{n-2}$ only), where

$$
\sum_{j=0}^{n-1} l_{i} \frac{\partial}{\partial x_{j}} \in \mathcal{H}
$$

${ }^{2}$ Notice in the diagram (5.27), the vertical $c_{0}^{*}$ is different from the horizontal $c_{0}^{*}$ because they are the pull-backs of different vector bundles over $\mathbf{P}^{n}$. Sorry for the abusing of notations. 
Because all $q_{2}, g_{k}$ are generic,

$$
\operatorname{Pr}\left(q_{2}\right), \operatorname{Pr}\left(g_{k}\right)
$$

are generic sections of $\mathcal{O}_{\mathbf{P}_{\text {sub }}^{1}}(2 n-4)$ and $\mathcal{O}_{\mathbf{P}_{\text {sub }}^{1}}(2 n-3)$ respectively.

Hence for the fixed $q_{2}, g_{k}$, the equation

$$
\sum_{i=1}^{2} l_{n-i}\left(x_{n-1}, x_{n-2}\right) \operatorname{Pr}\left(\frac{\partial q_{1}}{\partial x_{n-i}} q_{2}\right)+\sum_{k=0}^{n-3} l_{k}\left(x_{n-1}, x_{n-2}\right) \operatorname{Pr}\left(g_{k}\right)=0
$$

implies

$$
\sum_{i=1}^{2} l_{n-i}\left(x_{n-1}, x_{n-2}\right) \frac{\partial q_{1}}{\partial x_{n-i}}=0
$$

and $l_{k}=0, k=0, \cdots, n-3$. Thus the kernel of $\operatorname{Pr} \circ \xi$ is

$$
\left\{\left(l_{0}, \cdots, l_{n-1}\right): \sum_{i=1}^{2} l_{n-i}\left(x_{n-1}, x_{n-2}\right) \frac{\partial q_{1}}{\partial x_{n-i}}=0, l_{0}=\cdots=l_{n-3}=0\right\}
$$

which clearly has dimension 1 . Thus $\operatorname{dim}(\operatorname{image}(\operatorname{Pr} \circ \xi))=2 n-1$, which is the dimension of $H^{0}\left(\mathcal{O}_{\mathbf{P}^{1}}(h)\right)$. Hence $\operatorname{Pr} \circ \xi$ is surjective (onto $H^{0}\left(\mathcal{O}_{\mathbf{P}_{\text {sub }}^{1}}(h)\right)$ ). Thus

$$
\operatorname{image}\left(\nu^{s}\right) \supset \operatorname{image}\left(\nu^{s} \circ c_{0}^{*}\right)=c_{0}^{*}\left(H^{0}\left(\mathcal{O}_{\mathbf{P}_{\text {sub }}^{1}}(h)\right)\right) .
$$

By formula (5.23), we proved (5.17)

\section{REFERENCES}

[1] A. Albano and S. Katz, Lines on the Fermat quintic threefold and the infinitesimal generalized Hodge conjecture, Tran. of Amer. Math. Soc., 324 (1991), pp. 353-368,

[2] H. Clemens 1, Private letters, 2010.

[3] H. Clemens 2, Curves in generic hypersurfaces, Ann. Sci. École Norm. Sup., 19 (1986), pp. 629 636

[4] H. Clemens 3, Homological equivalence, modulo algebraic equivalence, is not finitely generated, Publ. Math IHES, 58 (1983), pp. 19-38

[5] H. Clemens 4, Cohomology and obstructions I: Geometry of formal Kuranishi theory, math.AG/9901084.

[6] H. Clemens 5, Cohomology and obstructions III: A variational form of the generalized Hodge conjecture on K-trivial threefolds, math.AG/9809127v4.

[7] S. Katz, On the finiteness of rational curves on quintic threefolds, Comp. Math., 60 (1986).

[8] G. Pacienza, Rational curves on general projective hypersurfaces, J. Algebraic Geometry, 12 (2003), pp. 471-476.

[9] C. Voisin, On a conjecture of Clemens on rational curves on hypersurfaces, J. Differential Geometry, 44 (1996), pp. 200-213.

[10] C. Voisin, A correction: "On a conjecture of Clemens on rational curves on hypersurfaces", J. Differential Geometry, 49 (1998), pp. 601-611. 